\title{
Crystal Structure of 1-(4-Benzylpiperazino)methyl-3-phenyl-1,2,4-triazole- 5-thione
}

\author{
Zarife Sibel GüL, ${ }^{* \dagger}$ Şamil IșıK,* Birsen Tozkoparan, ** and Mevlüt ERTAN** \\ *Department of Physics, Faculty of Arts and Sciences, Ondokuz Mayıs University, \\ Kurupelit, 55139 Samsun, Turkey \\ **Department of Pharmaceutical Chemistry Faculty of Pharmacy Hacettepe University, \\ 06100 Sihhiye, Ankara, Turkey
}

\begin{abstract}
The title compound, 1-(4-benzylpiperazino)methyl-3-phenyl-1,2,4-triazole-5-thione, $\mathrm{C}_{20} \mathrm{H}_{23} \mathrm{~N}_{5} \mathrm{~S}$, crystallizes in the monoclinic space group $P 2 / c$ with the following unit cell parameters: $a=18.6157(9) \AA, b=11.0013(5) \AA, c=$ $21.6202(14) \AA, \beta=120.561(4)^{\circ}$ and $V=3812.7(4) \AA^{3}$. The crystal structure was solved with a final $R=0.1045$ using 4996 independent reflections and contains two independent molecules in the asymmetric unit. Each independent molecule exists as part of an $\mathrm{N}-\mathrm{H} \cdots \mathrm{N}$ hydrogen-bonded centrosymmetric $\mathrm{R}_{2}{ }^{2}(18)$ dimer. An extensive three-dimensional network of $\mathrm{N}-\mathrm{H} \cdots \mathrm{N}, \mathrm{C}-\mathrm{H} \cdots \mathrm{N}$ and $\mathrm{C}-\mathrm{H} \cdots \mathrm{S}$ hydrogen bonds, with $\pi-\pi$ and $\pi$-ring interactions are responsible for crystal stabilization. Intermolecular hydrogen bonds and $\mathrm{C}-\mathrm{H} \cdots \pi$ interactions produce $\mathrm{R}_{2}{ }^{2}(10), \mathrm{R}_{3}{ }^{3}(19), \mathrm{R}_{4}{ }^{4}(26), \mathrm{R}_{6}{ }^{6}(37)$ and $\mathrm{R}_{8}{ }^{8}(54)$ rings.
\end{abstract}

(Received May 31, 2007; Accepted September 19, 2007; Published on web November 13, 2007)

Since the development of new analgesic/antiinflammatory compounds that are safer and as effective as opioids for the treatment of pain and inflammation continues to be a challenging problem in medicinal chemistry, our recent interest has focused on the synthesis and chemistry of this type of compound. We have synthesized various potential analgesicantiinflammatory substances derived from 1,2,4-triazole-5thiones. ${ }^{1}$ The title compound is one of the mannich derivatives of 1,2,4-triazole-5-thiones having analgesic antiinflammatory activity. The details of the concerned compound were published elsewhere. ${ }^{2}$ Here, we report on its crystal structure.

To a solution of 3-phenyl-1,2,4-triazole-5-thione (1.5 mmol)

Table 1 Crystal and experimental data

Formula: $\mathrm{C}_{20} \mathrm{H}_{23} \mathrm{~N}_{5} \mathrm{~S}$

Formula weight: 365.49

Crystal system: monoclinic

Space group: $P 2 / c \quad Z=8$

$a=18.6157(9) \AA$

$b=11.0013(5) \AA \quad \beta=120.561(4)^{\circ}$

$c=21.6202(14) \AA$

$V=3812.7(4) \AA^{3}$

Density $=1.273 \mathrm{~g} \mathrm{~cm}^{-1}$

No. of reflections used $=38736$

$2 \theta_{\max }=59.36^{\circ}$ Mo $K_{a}$

$R=0.1045$

$(\Delta / \sigma)_{\max }=0.000$

$(\Delta \rho)_{\max }=0.69{\mathrm{e} \AA^{-3}}^{-3}$

$(\Delta \rho)_{\min }=-0.35$ e $\AA^{-3}$

Measurement: STOE IPDS II

Program system: STOE X-RED

Structure determination: Direct methods

Refinement: full matrix

CCDC number: CCDC 648387

$\dagger$ To whom correspondence should be addressed.

E-mail: sgul@omu.edu.tr
Table 2 Atomic coordinates and temperature factors

\begin{tabular}{|c|c|c|c|c|}
\hline Atom & $x$ & $y$ & $z$ & $U_{\text {eq }}$ \\
\hline ClA & $0.3475(3)$ & $-0.2571(5)$ & $0.2101(3)$ & $0,0450(11)$ \\
\hline $\mathrm{C} 2 \mathrm{~A}$ & $0.3967(4)$ & $-0.3605(5)$ & $0.2353(3)$ & $0.0539(13)$ \\
\hline $\mathrm{C} 3 \mathrm{~A}$ & $0.3706(5)$ & $-0.4688(5)$ & $0.1981(4)$ & $0.0645(17)$ \\
\hline $\mathrm{C} 4 \mathrm{~A}$ & $0.2953(5)$ & $-0.4758(6)$ & $0.1346(4)$ & $0.0671(17)$ \\
\hline C5A & $0.2458(4)$ & $-0.3735(6)$ & $0.1089(4)$ & $0.0661(17)$ \\
\hline$C 6 A$ & $0.2710(4)$ & $-0.2664(5)$ & $0.1460(3)$ & $0.0572(14)$ \\
\hline C7A & $0.3741(3)$ & $-0.1409(5)$ & $0.2487(3)$ & $0.0436(11)$ \\
\hline $\mathrm{C} 8 \mathrm{~A}$ & $0.3790(3)$ & $0.0508(5)$ & $0.2700(3)$ & $0.0463(11)$ \\
\hline $\mathrm{C} 9 \mathrm{~A}$ & $0.4856(4)$ & $0.0375(6)$ & $0.4038(3)$ & $0.0530(13)$ \\
\hline $\mathrm{C} 10 \mathrm{~A}$ & $0.5792(4)$ & $0.1541(6)$ & $0.3796(3)$ & $0.0561(14)$ \\
\hline $\mathrm{CllA}$ & $0.6706(4)$ & $0.1869(5)$ & $0.4114(4)$ & $0.0574(14)$ \\
\hline $\mathrm{Cl} 2 \mathrm{~A}$ & $0.6194(4)$ & $-0.0474(5)$ & $0.4295(3)$ & $0.0530(13)$ \\
\hline $\mathrm{C} 13 \mathrm{~A}$ & $0.7109(4)$ & $-0.0166(6)$ & $0.4612(3)$ & $0.0567(14)$ \\
\hline C14A & $0.8101(4)$ & $0.1113(6)$ & $0.4451(4)$ & $0.0630(16)$ \\
\hline $\mathrm{C} 15 \mathrm{~A}$ & $0.8591(3)$ & $0.0163(5)$ & $0.4332(3)$ & $0,0511(13)$ \\
\hline $\mathrm{C} 16 \mathrm{~A}$ & $0.9043(4)$ & $-0.0710(6)$ & $0.4845(3)$ & $0.0617(16)$ \\
\hline C17A & $0.9507(4)$ & $-0.1556(6)$ & $0.4744(4)$ & $0.0703(19)$ \\
\hline C18A & $0.9544(4)$ & $-0.1553(7)$ & $0.4130(4)$ & $0.0715(19)$ \\
\hline C19A & $0.9095(4)$ & $-0.0699(7)$ & $0.3598(3)$ & $0.0687(18)$ \\
\hline $\mathrm{C} 20 \mathrm{~A}$ & $0.8614(4)$ & $0.0172(6)$ & $0.3695(3)$ & $0.0571(14)$ \\
\hline N1A & $0.4317(3)$ & $-0.0118(4)$ & $0.3309(2)$ & $0.0480(10)$ \\
\hline $\mathrm{N} 2 \mathrm{~A}$ & $0.4289(3)$ & $-0.1335(4)$ & $0.3177(2)$ & $0.0479(10)$ \\
\hline $\mathrm{N} 3 \mathrm{~A}$ & $0.3427(3)$ & $-0.0320(4)$ & $0.2174(2)$ & $0.0453(10)$ \\
\hline N4A & $0.5715(3)$ & $0.0614(5)$ & $0.4253(3)$ & $0.0534(11)$ \\
\hline N5A & $0.7199(3)$ & $0.0774(4)$ & $0.4156(3)$ & $0.0507(11)$ \\
\hline \$2 & $0.36063(11)$ & $0.20316(13)$ & $0.26394(9)$ & $0.0626(5)$ \\
\hline $\mathrm{ClB}$ & $0.1396(3)$ & $0.1817(5)$ & $0.3597(3)$ & $0.0458(11)$ \\
\hline $\mathrm{C} 2 \mathrm{~B}$ & $0.0909(4)$ & $0.0778(5)$ & $0.3393(3)$ & $0.0567(14)$ \\
\hline $\mathrm{C} 3 \mathrm{~B}$ & $0.1217(5)$ & $-0.0296(6)$ & $0.3304(4)$ & $0.0693(18)$ \\
\hline $\mathrm{C} 4 \mathrm{~B}$ & $0.2004(5)$ & $-0.0361(6)$ & $0.3402(4)$ & $0.0720(19)$ \\
\hline $\mathrm{C} 5 \mathrm{~B}$ & $0.2476(5)$ & $0.0673(6)$ & $0.3587(4)$ & $0.0671(17)$ \\
\hline $\mathrm{C} 6 \mathrm{~B}$ & $0.2194(4)$ & $0.1748(5)$ & $0.3700(4)$ & $0.0562(14)$ \\
\hline $\mathrm{C} 7 \mathrm{~B}$ & $0.1091(3)$ & $0.2977(5)$ & $0.3726(3)$ & $0.0446(11)$ \\
\hline $\mathrm{C} 8 \mathrm{~B}$ & $0.1071(3)$ & $0.4842(5)$ & $0.3998(3)$ & $0.0489(12)$ \\
\hline $\mathrm{C9B}$ & $-0.0282(4)$ & $0.4718(6)$ & $0.4000(3)$ & $0.0537(13)$ \\
\hline $\mathrm{Cl} 0 \mathrm{~B}$ & $-0.0883(4)$ & $0.5784(5)$ & $0.2849(3)$ & $0.0555(14)$ \\
\hline $\mathrm{C} 11 \mathrm{~B}$ & $-0.1703(4)$ & $0.6248(5)$ & $0.2249(3)$ & $0.0603(16)$ \\
\hline $\mathrm{C} 12 \mathrm{~B}$ & $-0.1514(3)$ & $0.3937(5)$ & $0.2905(3)$ & $0.0506(\mathrm{l} 3)$ \\
\hline $\mathrm{C} 13 \mathrm{~B}$ & $-0.2357(4)$ & $0.4367(6)$ & $0.2302(3)$ & $0.0527(13)$ \\
\hline C14B & $-0.3080(4)$ & $0.5726(6)$ & $0.1245(3)$ & $0.0621(16)$ \\
\hline $\mathrm{C} 15 \mathrm{~B}$ & $-0.3606(4)$ & $0.4803(5)$ & $0.0706(3)$ & $0.0502(13)$ \\
\hline $\mathrm{C} 16 \mathrm{~B}$ & $-0.3531(4)$ & $0.4577(6)$ & $0.0106(3)$ & $0.0592(15)$ \\
\hline $\mathrm{C} 17 \mathrm{~B}$ & $-0.4068(5)$ & $0.3771(7)$ & $-0.0424(4)$ & $0.0697(18)$ \\
\hline C18B & $-0.4654(4)$ & $0.3170(6)$ & $-0.0349(4)$ & $0.0680(18)$ \\
\hline $\mathrm{C} 19 \mathrm{~B}$ & $-0.4743(4)$ & $0.3371(6)$ & $0.0238(3)$ & $0.0612(16)$ \\
\hline $\mathrm{C} 20 \mathrm{~B}$ & $-0.4220(4)$ & $0.4172(6)$ & $0.0764(3)$ & $0.0568(14)$ \\
\hline $\mathrm{N} 1 \mathrm{~B}$ & $0.0415(3)$ & $0.4236(4)$ & $0.3960(2)$ & $0.0505(11)$ \\
\hline N2B & $0.0420(3)$ & $0.3026(4)$ & $0.3782(2)$ & $0.0504(11)$ \\
\hline N3B & $0.1491(3)$ & $0.4048(4)$ & $0.3839(2)$ & $0.0480(10)$ \\
\hline $\mathrm{N} 4 \mathrm{~B}$ & $-0.1029(3)$ & $0.4981(4)$ & $0.3314(2)$ & $0.0487(10)$ \\
\hline N5B & $-0.2253(3)$ & $0.5236(4)$ & $0.1821(2)$ & $0.0465(10)$ \\
\hline S1 & $0.13098(11)$ & $0.63352(15)$ & $0.42328(11)$ & $0.0699(5)$ \\
\hline
\end{tabular}




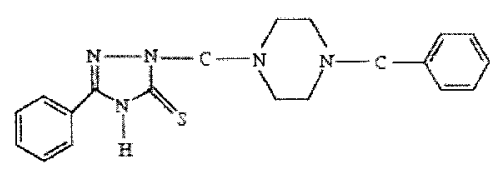

Fig. 1 The schematic diagram of the title compound.

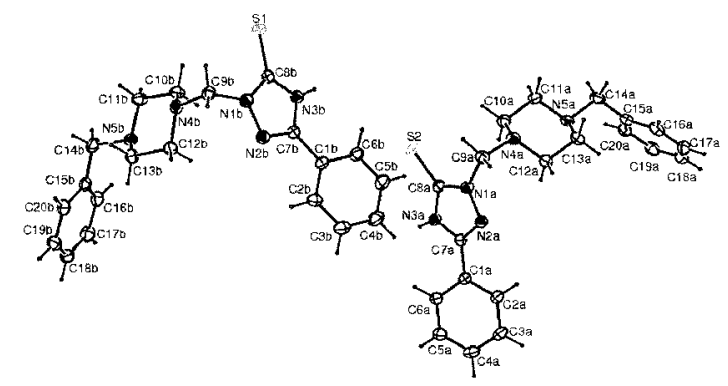

Fig. 2 The molecular structure of (I), showing the atom-numbering scheme. Displacement ellipsoids are drawn at the $40 \%$ probability.

Table 3 Selected geometric parameters $(\AA)$

\begin{tabular}{lll}
\hline C7A N2A 1.313(7) & C8A N3A 1.341(7) & C8A S2 1.702(6) \\
N1A N2A 1.364(6) & C7B N2B 1.318(7) & C8B N3B 1.329(7) \\
C8B S1 1.710(6) & N1B N2B 1.387(6) & \\
\hline
\end{tabular}

in $\mathrm{EtOH}(20 \mathrm{~mL})$ was added an equimolar quantity of benzylpiperazine, and then $35 \%$ aqueous formaldehyde $(0.3$ $\mathrm{mL})$. The reaction mixture was refluxed over a water-bath for 2 $\mathrm{h}$ and cooled to room temperature. The resulting precipitate was filtered and recrystallized from ethanol.

The structure was solved by direct methods with SHELXS-973 and refined by full-matrix least-squares procedures on $F^{2}$, using the program SHELXL-97. ${ }^{3}$ All non-hydrogen atoms were refined anisotropically. All hydrogen atom positions were refined using a riding model. Since the quality of the examined crystal was poor, the index values of $R(0.1045)$ and $w R$ (0.3612) are not good enough for a crystallographic solution.

The molecular structure of (Fig. 1) has two crystallograhically independent molecules in the asymmetric unit (Fig. 2); selected bond lengths are given in Table 3 . The compound crystallizes in the space group $P 2 / c$ with $Z^{\prime}=2$, and the hydrogen bonding was analyzed with the aid of PLATON. ${ }^{4}$ The N2A-C7A and N2B-C7B bond lengths are indicative of a significant doublebond character, respectively (Table 3 ).

Atom $\mathrm{N} 3 \mathrm{~A}$ in the reference molecule at $(x, y, z)$ acts as a hydrogen-bond donor via $\mathrm{H} 3 \mathrm{AA}$, respectively, to atom $\mathrm{N} 5 \mathrm{~A}^{\mathrm{i}}$, and thus forms a centrosymmetric $\mathrm{R}_{2}{ }^{2}(18)[\mathrm{A}] .^{5}$ Similarly, atom $\mathrm{N} 3 \mathrm{~B}$ acts as hydrogen-bond donor via $\mathrm{H} 3 \mathrm{BB}$ to $\mathrm{N} 5 \mathrm{~B}^{\mathrm{ii}}$, so as to form a second centrosymmetric ring motif, this time of the $\mathrm{R}_{2}{ }^{2}(18)$ [B] type. The propagation of $\mathrm{C}-\mathrm{H} \cdots \mathrm{S}$ hydrogen-bonds thus forms a chain of edge-fused $\mathrm{R}_{2}{ }^{2}(18)$ [A] and $\mathrm{R}_{2}{ }^{2}(18)$ [B] rings running parallel to [100], generated by translation. The interlinked $\mathrm{C}_{3}{ }^{3}(12)$ antiparallel chains along the $a$-axis are formed through $\mathrm{C} 13 \mathrm{~B}-\mathrm{H} 133 \cdots \mathrm{S} 2^{\mathrm{ii}}$, N3B-H3BB $\cdots \mathrm{N} 5 \mathrm{~B}^{\mathrm{ii}}$ and $\mathrm{C} 13 \mathrm{~B}^{\mathrm{ii}}-\mathrm{H} 133^{\mathrm{ii} \ldots \mathrm{S} 2}$ interactions [symmetry codes in Table 4]. Similarly, interlinked $\mathrm{C}_{3}{ }^{3}(22)$ antiparallel chains along the $c$ axis are formed through C18B-H18B $\cdots \mathrm{N} 2 \mathrm{~A}^{\text {iii }}$, $\mathrm{N} 3 \mathrm{~B}-\mathrm{H} 3 \mathrm{BB} \cdots \mathrm{N} 5 \mathrm{~B}^{\mathrm{ii}}$ and $\mathrm{C} 18 \mathrm{~B}^{\mathrm{ii}-\mathrm{H}} 18 \mathrm{~B}^{\mathrm{ii} \ldots \mathrm{N}} \mathrm{A}^{\mathrm{vii}}$ [symmetry code: (vii) $=1-x,-y, 1-z]$ interactions. Hence, the combination of the $\mathrm{C}_{3}{ }^{3}(12), \mathrm{C}_{3}{ }^{3}(22)$ and $\mathrm{R}_{2}{ }^{2}(18)$ motifs generates a sheet containing $\mathrm{R}_{2}{ }^{2}(18), \mathrm{R}_{4}{ }^{4}(26)$ and $\mathrm{R}_{8}{ }^{8}(54)$ rings, alternating in a checkerboard fashion (Fig. 3).

In the extended structure, there are also $\pi$ - $\pi$ and $\pi$-ring

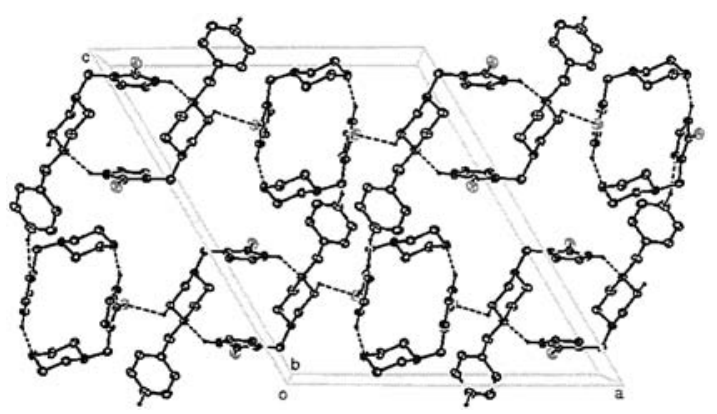

Fig. 3 A stereo view of part of the crystal structure of (I), showing the formation of a hydrogen-bonded sheet built from $\mathrm{R}_{2}{ }^{2}(18), \mathrm{R}_{4}{ }^{4}(26)$ and $\mathrm{R}_{8}{ }^{8}(54)$ rings. $\mathrm{H}$ atoms not involved in these interactions and phenyl rings have been omitted for clarity.

Table 4 Hydrogen-bond geometry $(\AA)$

\begin{tabular}{|c|c|c|c|c|}
\hline $\mathrm{D}-\mathrm{H} \cdots \mathrm{A}$ & $\mathrm{D}-\mathrm{H}$ & $\mathrm{H} \cdots \mathrm{A}$ & $\mathrm{D} \cdots \mathrm{A}$ & $\mathrm{D}-\mathrm{H} \cdots \mathrm{A}$ \\
\hline N3A-H3AA ...N5A & 0.86 & 2.01 & $2.769(6)$ & 146 \\
\hline $\mathrm{N} 3 \mathrm{~B}-\mathrm{H} 3 \mathrm{BB} \ldots \mathrm{N} 5 \mathrm{~B}^{\mathrm{ii}}$ & 0.86 & 2.10 & $2.794(7)$ & 138 \\
\hline $\mathrm{C} 13 \mathrm{~B}-\mathrm{H} 133 \ldots \mathrm{S} 2^{\mathrm{iz}}$ & 0.97 & 2.83 & $3.511(6)$ & 128 \\
\hline $\mathrm{C} 18 \mathrm{~B}-\mathrm{H} 18 \mathrm{~B} \ldots \mathrm{N} 2 \mathrm{~A}^{\mathrm{Li}}$ & 0.93 & 2.53 & $3.425(8)$ & 163 \\
\hline $\mathrm{CGA}-\mathrm{H} 6 \mathrm{~A} \ldots \mathrm{Cg} 3^{\mathrm{i}}$ & 0.93 & 2.71 & $3.581(8)$ & 156 \\
\hline $\mathrm{C} 13 \mathrm{~A}-\mathrm{H} 131 \ldots \mathrm{Cg}^{2 \mathrm{ir}}$ & 0.97 & 2.80 & $3.576(6)$ & 138 \\
\hline C6B-H6B...Cg4 & 0.93 & 3.14 & $4.009(7)$ & 157 \\
\hline $\mathrm{C} 13 \mathrm{~B}-\mathrm{H} 134 \ldots \mathrm{Cg} 1^{\mathrm{i}}$ & 0.97 & 2.93 & $3.880(7)$ & 166 \\
\hline C17B-H17B $\mathrm{Cg} 1^{\mathrm{vi}}$ & 0.93 & 3.00 & $3.870(9)$ & 157 \\
\hline
\end{tabular}

$\mathrm{Cg} 1=\mathrm{C} 1 \mathrm{~A}-\mathrm{C} 6 \mathrm{~A} ; \mathrm{Cg} 2=\mathrm{C} 1 \mathrm{~B}-\mathrm{C} 6 \mathrm{~B} ; \mathrm{Cg} 3=\mathrm{C} 15 \mathrm{~A}-\mathrm{C} 20 \mathrm{~A} ; \mathrm{Cg} 4=\mathrm{C} 15 \mathrm{~B}-$ C20B (i $=-x+1, y,-z+1 / 2),(\mathrm{ii}=-x, y,-z+1 / 2),(\mathrm{iii}=x-1,-y, z-1 / 2)$, (iv $=-x,-y+1,-z),(\mathrm{v}=-x, y,-z+1 / 2),(\mathrm{vi}=-x+1,-y+1,-z+1)$

interactions. An intermolecular $\pi$ - $\pi$ contact occurs between the two symmetry-related phenyl rings $(\mathrm{Cg} 3)$ of neighboring molecules. Ring $\mathrm{Cg} 3$ is oriented in such a way that the perpendicular distance from $\mathrm{Cg} 3$ to $\mathrm{Cg} 3^{\text {viii }}$ is $3.347 \AA$ [symmetry code: (viii) $=-x, 2-y,-z]$. The distance between the ring centroids is $3.721(4) \AA$. Similarly, the other two symmetryrelated phenyl rings, $\mathrm{Cg} 4$ and $\mathrm{Cg} 4^{\text {vi }}$, also form an intermolecular $\pi-\pi$ contact. Rings $\mathrm{Cg} 4$ and $\mathrm{Cg} 4^{\text {vi }}$ are oriented in such a way that the perpendicular distance from $\mathrm{Cg} 4$ to $\mathrm{Cg} 4^{\text {vi }}$ is $3.295 \AA$. The distance between the ring centroids is 3.674(4) $\AA$. The compound also contains five intermolecular $\mathrm{C}-\mathrm{H} \cdots \pi$ interactions between rings $\mathrm{Cg} 1, \mathrm{Cg} 2, \mathrm{Cg} 3$ and $\mathrm{Cg} 4$. The details of these interactions are given in Table 4. Intermolecular hydrogen bonds and $\mathrm{C}-\mathrm{H} \cdots \pi$ interactions produce $\mathrm{R}_{2}{ }^{2}(10), \mathrm{R}_{3}{ }^{3}(19)$ and $\mathrm{R}_{6}{ }^{6}(37)$ rings.

\section{References}

1. B. Tozkoparan, G. Ayhan Kilcigil, R. Ertan, M. Ertan, P. Kelicen, and R. Demirdamar, Arzneim. Forsch./Drug Res., 1999, 49, 1006.

2. B. Tozkoparan, E. Küpeli, E. Yeşilada, Ş. Isık, M. Özalp, and M. Ertan, Arzneim. Forsch./Drug Res., 2005, 55, 533.

3. G. M. Sheldrick, SHELXS97 and SHELXL97, 1997, University of Göttingen, Germany.

4. A. L. Spek, J. Appl. Cryst., 2003, 36, 7.

5. J. Bernstein, R. E. Davis, L. Shimoni, and N. L. Chang, Angew. Chem. Int. Ed. Engl., 1995, 34, 1555. 